\title{
Magnetic impurity effect on the entanglement in the Ising model
}

\author{
Junpeng Cao ${ }^{1,2}$, Z Z Sun ${ }^{1}$, S Yin ${ }^{1}$, Yupeng Wang ${ }^{2,3}$ and X R Wang ${ }^{1}$ \\ ${ }^{1}$ Department of Physics, The Hong Kong University of Science and Technology, \\ Hong Kong SAR, People's Republic of China \\ ${ }^{2}$ Institute of Physics, Chinese Academy of Sciences, Beijing 100080, People's Republic of China \\ ${ }^{3}$ International Center for Quantum Structures, Chinese Academy of Sciences, Beijing 100080, \\ People's Republic of China \\ E-mail:phxwan@ust.hk
}

Received 12 July 2004, in final form 4 January 2005

Published 9 March 2005

Online at stacks.iop.org/JPhysA/38/2579

\begin{abstract}
We study the effect of magnetic impurity on the entanglement in the three-site Ising model with both periodic and open boundary conditions. We obtain the analytic expressions for entanglement and show that the ground state and excited states of the system possess remarkable entanglement properties. We find that the entanglement between two specific spins for a given state depends on the sites where the impurities are located. We also discuss a relation of our results with some aspects of quantum phase transitions in the transverse Ising model.
\end{abstract}

PACS numbers: 03.67.Mn, 03.65.Ud, 75.10.Jm

(Some figures in this article are in colour only in the electronic version)

\section{Introduction}

Quantum entanglement plays a central role in quantum information and quantum computation. It is an important resource for processing quantum information [1]. With quantum entanglement, one can perform quantum teleportation [2], super-dense coding [3], quantum cryptography [4], etc. Over the past years much effort has been put into studying the entanglement in various quantum spin systems at finite temperature. The thermal entanglement was introduced and analysed within the Ising model in a transverse field [5], the Heisenberg XXX [6], XX [7] and XXZ models [8]. The enhanced thermal entanglement in an anisotropic three-site Heisenberg XYZ chain was also observed [9]. The state of the system at thermal equilibrium is represented by the density operator $\rho(T)=\exp \left(-H /\left(k_{B} T\right)\right) / Z$, where $Z=\operatorname{tr}\left[\exp \left(-H /\left(k_{B} T\right)\right)\right]$ is the partition function and $k_{B}$ is the Boltzmann constant. The entanglement at the thermal equilibrium state is the thermal entanglement. 
Entanglement can play a crucial role in quantum phase transitions [10]. Osterloh et al studied the scaling of entanglement close to the quantum phase transitions and showed that the pairwise quantum entanglement between nearest neighbour sites and next nearest neighbour sites of the ground state of XY spin chain displays a peak either near or at the critical point of quantum phase transitions [11]. Similar results are also obtained and generalized in [12]. Vidal et al pointed out that entanglement of the ground state of XXZ and XY spin chains at the critical point is analogous to that of entropy in conformal field theories [13]. Osenda et al [14] studied the impurities entanglement in the quantum XY model. Some related work was also done for various models [15-18]. Quantifying entanglement is a very interesting issue in modern academic research. The strongly entangled systems exhibit complicated behaviour which is difficult to quantify. The Ising model is very important in statistical mechanics and condensed matter physics. Many materials, such as $\mathrm{CsCoCl}_{3},\left(\mathrm{NH}_{4}\right)_{2} \mathrm{MnF}_{5}$ and $\mathrm{FeCl}_{2} \mathrm{Py}_{2}$, have this kind of exchanging interaction. The transverse Ising model is a wonderful example to study the quantum phase transitions. In this paper, we study and quantify the entanglement in the three-site Ising model with impurities and transverse fields.

The structure of this paper is as follows. In section 2, some measures of entanglement are outlined. We study the entanglement in the Ising model with one impurity in section 3 . The two-impurity effect on the entanglement in the system is studied in section 4. In section 5, some results of the transverse Ising model are given. In section 6, we discuss the entanglement properties in the Ising model with two boundary impurities. In section 7, we discuss the system with two neighbour impurities using the free boundary conditions. Section 8 concludes the paper.

\section{The measures of entanglement}

Recall some measures of entanglement. Let $\rho_{12}$ be the density matrix of two qubits and it can denote either a pure or a mixed state. The concurrence [19] is defined as

$$
C_{12}=\max \left\{\lambda_{1}-\lambda_{2}-\lambda_{3}-\lambda_{4}, 0\right\}
$$

where the quantities $\lambda_{i}$ are the square roots of the eigenvalues of the operator $R_{12}=$ $\rho_{12}\left(\sigma_{1}^{y} \otimes \sigma_{2}^{y}\right) \rho_{12}^{*}\left(\sigma_{1}^{y} \otimes \sigma_{2}^{y}\right)$ in descending order. The eigenvalues of $R_{12}$ are real and non-negative even though $R_{12}$ is not necessarily Hermitian. The values of the concurrence monotonically increase and range from 0 , for an unentangled state, to 1 , for a maximally entangled state, and can describe the degree of entanglement. In this method, the standard basis, $\{|\uparrow \uparrow\rangle,|\uparrow \downarrow\rangle,|\downarrow \uparrow\rangle,|\downarrow \downarrow\rangle\}$, must be used. Tangle [20] is also a measure of entanglement and is defined only for pure states. Consider a multi-particle system where one of the subsystems, denoted as $j$, is a qubit. The entanglement between the qubit $j$ and the rest of the qubits, denoted as $\bar{j}$, is defined as tangle,

$$
C_{j \bar{j}}=4 \operatorname{Det} \rho_{j}=2\left(1-\operatorname{tr} \rho_{j}^{2}\right)
$$

where $\rho_{j}$ is the reduced density matrix of the qubit $j, \rho_{j}=\operatorname{tr}_{\bar{j}}|\psi\rangle\langle\psi|$, and $\operatorname{tr}_{\bar{j}}$ stands for tracing over all qubits except the $j$ th qubit. As usual for entanglement measures, the tangle ranges from 0 (no entanglement) to 1 , when two qubits are maximally entangled. With concurrence and tangle, the residual entanglement three-qubit pure state is defined as $C_{i j k}=C_{i \bar{i}}-C_{i j}^{2}-C_{i k}^{2}$. The global entanglement [21] of the multi-particle system is defined as

$$
Q(|\psi\rangle)=2\left(1-\frac{1}{n} \sum_{k=1}^{n} \operatorname{tr}\left(\rho_{k}^{2}\right)\right)
$$

which means that the multi-particle entanglement is an average over the entanglement of each qubit with the rest of the system. The measure $Q$ has nice properties: (i) $0 \leqslant Q \leqslant 1 . Q=0$ if 
and only if $|\psi\rangle$ is a product state and $Q=1$ for maximally entangled states, (ii) $Q$ is invariant under local unitaries, as a good measure should be.

\section{Entanglement in the Ising model with one magnetic impurity}

We first consider a three-site Ising model with a magnetic impurity on the third site,

$$
H=-J\left(\sigma_{1}^{x} \sigma_{2}^{x}+\sigma_{2}^{x} \sigma_{3}^{x}+\sigma_{3}^{x} \sigma_{1}^{x}\right)+B \sigma_{3}^{z}
$$

where $\sigma_{i}^{\alpha}(\alpha=x, z)$ are the Pauli matrices of the $i$ th site, $J$ is the exchanging interaction constant and $B$ is the magnetic impurity and is assumed larger than zero, which also means an external magnetic field on the third site. We adopt periodic boundary conditions and therefore the system is a three-qubit Ising ring with an external magnetic field on one of the sites. In order to study pairwise entanglement, the first step is to obtain all the eigenvalues and eigenstates of the Hamiltonian (4). By solving the time-independent Schrödinger equation, we obtain the eigenvalues of the system

$$
\begin{array}{ll}
E_{1}=E_{5}=-J-\sqrt{B^{2}+4 J^{2}} & E_{2}=E_{6}=-J+\sqrt{B^{2}+4 J^{2}} \\
E_{3}=E_{7}=J+B & E_{4}=E_{8}=J-B .
\end{array}
$$

The corresponding eigenstates are

$$
\begin{array}{llc}
\left|\psi_{i}\right\rangle & =k_{i}\left(|001\rangle+b_{i}|010\rangle+b_{i}|100\rangle+|111\rangle\right) & i=1,2 \\
\left|\psi_{3}\right\rangle & =(|001\rangle-|111\rangle) / \sqrt{2} \quad\left|\psi_{4}\right\rangle=(|010\rangle-|100\rangle) / \sqrt{2} \\
\left|\psi_{j}\right\rangle & =k_{j}\left(|110\rangle+b_{j}|011\rangle+b_{j}|101\rangle+|000\rangle\right) & j=5,6 \\
\left|\psi_{7}\right\rangle & =(|011\rangle-|101\rangle) / \sqrt{2} \quad\left|\psi_{8}\right\rangle=(|000\rangle-|110\rangle) / \sqrt{2}
\end{array}
$$

where $b_{1,2}=\left[B \pm\left(B^{2}+4 J^{2}\right)^{1 / 2}\right] /(2 J), b_{5,6}=\left[-B \pm\left(B^{2}+4 J^{2}\right)^{1 / 2}\right] /(2 J)$ and $k_{i, j}$ are the normalized coefficients.

The ground state is the linear combination of states $\left|\psi_{1}\right\rangle$ and $\left|\psi_{5}\right\rangle$ if $J>0$. The ground state is twofold degenerate. The thermal ground state is a mixed state and has the probabilities of one half occupying $\left|\psi_{1}\right\rangle$ and one half occupying $\left|\psi_{5}\right\rangle$. The pairwise entanglement at the thermal ground state is $C_{12}=C_{23}=C_{13}=0$. Let us consider two limit cases: (i) the ground state is $\left|\psi_{1}\right\rangle$ and (ii) the ground state is $\left|\psi_{5}\right\rangle$. In case (i), the entanglement of pairs 1 and 2 is $C_{12}=2 k_{1}^{2}\left|1-b_{1}^{2}\right|$ and the pairwise entanglement of both $(1,3)$ and $(2,3)$ is zero, $C_{13}=C_{23}=0$. We also have $C_{1 \overline{1}}=C_{2 \overline{2}}=1$, which means that site 1 (or 2) is maximally entangled with the subsystem consisting of sites 2 (or 1 ) and 3 , and $C_{3 \overline{3}}=16 k_{1}^{4} b_{1}^{2}$. The residual entanglement of the system is equal to the tangle between site 3 and the rest of the other sites. One can prove $C_{123}=C_{213}$ exactly, which means that $C_{123}$ is independent of permutations of sites and is a proper measure of entanglement. The global entanglement of the whole system is $Q\left(\left|\psi_{1}\right\rangle\right)=\left(2+16 k_{1}^{4} b_{1}^{4}\right) / 3$. The entanglement properties are clearly shown in figure 1. In case (ii), one can obtain the results using the same methods. While if $J<0, E_{4}$ and $E_{8}$ are the lowest energies. The thermal ground state has the probabilities of one half occupying $\left|\psi_{4}\right\rangle$ and one half occupying $\left|\psi_{8}\right\rangle$. The pairwise entanglement at the thermal ground state is $C_{12}=1, C_{23}=C_{13}=0$. If the ground state is $\left|\psi_{4}\right\rangle$, we have $C_{12}=1, C_{23}=C_{13}=0, C_{1 \overline{1}}=C_{2 \overline{2}}=1, C_{3 \overline{3}}=0, C_{123}=0$ and $Q\left(\left|\psi_{4}\right\rangle\right)=2 / 3$. These results are also valid for the case when the ground state is $\left|\psi_{8}\right\rangle$.

We now derive the concurrence for any pair of systems at finite temperature. The thermal entanglement between sites 1 and 3 is the same as that between sites 2 and 3 


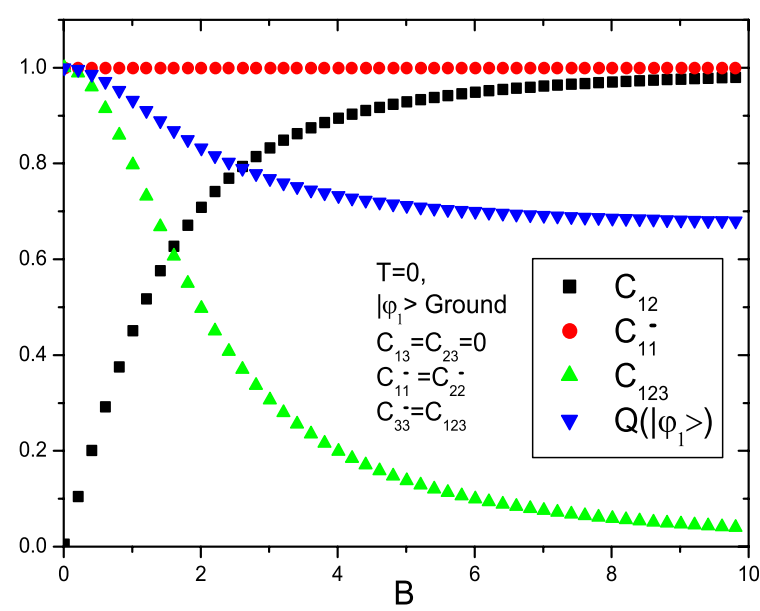

Figure 1. The ground state entanglement in the Ising model with single impurity. Here $J=1.0$. The concurrence $C_{12}$ increases with increasing magnitude of the magnetic impurity. The tangle, residual entanglement and global entanglement are zero if $B=0$ and reach their maxima immediately if there exists infinitesimal magnetic impurity. Then, the tangles $C_{1 \overline{1}}$ and $C_{2 \overline{2}}$ remain unchanged, while the residual and global entanglements decrease with increasing magnitude of impurity.

due to the symmetry. The state at thermal equilibrium is described by the density matrix $\rho(T)=\sum_{k=1}^{8} \exp \left(-\beta E_{k}\right)\left|\psi_{k}\right\rangle\left\langle\psi_{k}\right| / Z$, where $\beta=1 / T$ and the Boltzmann constant is set to 1 . The partition function is $Z=\sum_{i=1}^{8} \exp \left(-\beta E_{i}\right)$. The reduced density matrices $\rho_{12}(T)=\operatorname{tr}_{3} \rho(T)$ and $\rho_{13}(T)=\operatorname{tr}_{2} \rho(T)$, where $\operatorname{tr}_{i}$ denotes the tracing over the $i$ th site, take the following form:

$$
\rho_{12}=\frac{1}{Z}\left(\begin{array}{cccc}
A & & & D \\
& B & C & \\
& C & B & \\
D & & & A
\end{array}\right) \quad \rho_{13}=\frac{1}{Z}\left(\begin{array}{cccc}
\tilde{A} & & & \tilde{C} \\
& \tilde{B} & \tilde{C} & \\
& \tilde{C} & \tilde{A} & \\
\tilde{C} & & & \tilde{B}
\end{array}\right)
$$

The nonzero matrix elements of $\rho_{12}$ are given by $A=\sum_{i} k_{i}^{2} \mathrm{e}^{-\beta E_{i}}+0.5 \mathrm{e}^{-\beta E_{3}}+0.5 \mathrm{e}^{-\beta E_{4}}, B=$ $\sum_{i} k_{i}^{2} b_{i}^{2} \mathrm{e}^{-\beta E_{i}}+0.5 \mathrm{e}^{-\beta E_{3}}+0.5 \mathrm{e}^{-\beta E_{4}}, C=\sum_{i} k_{i}^{2} b_{i}^{2} \mathrm{e}^{-\beta E_{i}}-0.5 \mathrm{e}^{-\beta E_{3}}+0.5 \mathrm{e}^{-\beta E_{4}}, D=$ $\sum_{i} k_{i}^{2} \mathrm{e}^{-\beta E_{i}}-0.5 \mathrm{e}^{-\beta E_{3}}+0.5 \mathrm{e}^{-\beta E_{4}}$, where $i=1,2,5,6$. For $\rho_{13}$, we have $\tilde{A}=\left(k_{1}^{2} b_{1}^{2}+\right.$ $\left.k_{5}^{2}\right) \mathrm{e}^{-\beta E_{1}}+\left(k_{2}^{2} b_{2}^{2}+k_{6}^{2}\right) \mathrm{e}^{-\beta E_{2}}+\mathrm{e}^{-\beta E_{4}}, \tilde{B}=\left(k_{1}^{2}+k_{5}^{2} b_{5}^{2}\right) \mathrm{e}^{-\beta E_{1}}+\left(k_{2}^{2}+k_{6}^{2} b_{6}^{2}\right) \mathrm{e}^{-\beta E_{2}}+\mathrm{e}^{-\beta E_{3}}, \tilde{C}=$ $\left(k_{1}^{2} b_{1}+k_{5}^{2} b_{5}\right) \mathrm{e}^{-\beta E_{1}}+\left(k_{2}^{2} b_{2}+k_{6}^{2} b_{6}\right) \mathrm{e}^{-\beta E_{2}}$. One can easily prove $C_{12}=C_{13}=C_{23}=0$, which means that any pairwise thermal entanglement is zero. However, it does not mean that the system has no entanglement; the impurity can lead to entanglement of site $i$ with the rest of the other sites and global entanglement of the whole system. Note that at finite temperature, all excited states have contributions to the thermal entanglement. The thermal equilibrium state is a mixed state. The concurrence of two-qubit mixed state $\rho$ is defined to be a minimum over all possible pure-state decompositions of $\rho$ [22]. For a given mixed state $\left\{p_{i},\left|\psi_{i}\right\rangle\right\}$, the concurrences of different pure states of the mixed state may cancel each other [5]. These lead to zero pairwise thermal entanglement. One can check that the pairwise entanglement, tangle, residual entanglement and global entanglement of every excited state are not equal to zero. 


\section{Entanglement in the Ising model with two magnetic impurities}

To show the entanglement properties caused by impurities clearly, let us further consider the Ising ring with two magnetic impurities. The Hamiltonian is

$$
H=-J\left(\sigma_{1}^{x} \sigma_{2}^{x}+\sigma_{2}^{x} \sigma_{3}^{x}+\sigma_{3}^{x} \sigma_{1}^{x}\right)+B \sigma_{1}^{z}+B \sigma_{3}^{z} .
$$

One can easily obtain the eigenvalues of system (7) as

$$
\begin{array}{ll}
E_{1}=E_{5}=J & E_{2}=E_{6}=P J+Q J-J / 3 \\
E_{3}=E_{7}=w P J+w^{2} Q J-J / 3 & E_{4}=E_{8}=w^{2} P J+w Q J-J / 3
\end{array}
$$

where $P=\left(-q / 2+\Delta^{1 / 2}\right)^{1 / 3}, Q=\left(-q / 2-\Delta^{1 / 2}\right)^{1 / 3}, \Delta=(q / 2)^{2}+(p / 3)^{3}, q=[128-72$ $\left.(B / J)^{2}\right] / 27, p=-\left[12(B / J)^{2}+16\right] / 3, w=(-1+\sqrt{-3}) / 2$. The corresponding eigenstates are

$$
\begin{aligned}
& \left|\psi_{1}\right\rangle=(|011\rangle-|110\rangle) / \sqrt{2} \quad\left|\psi_{5}\right\rangle=(|100\rangle-|001\rangle) / \sqrt{2} \\
& \left|\psi_{j}\right\rangle=k_{j}\left(|000\rangle+b_{j}|011\rangle+a_{j}|101\rangle+b_{j}|110\rangle\right) \\
& \left|\psi_{l}\right\rangle=k_{l}\left(|111\rangle+b_{l}|100\rangle+a_{l}|010\rangle+b_{l}|001\rangle\right)
\end{aligned}
$$

where $a_{j}=\left[-\left(E_{j}+J\right)\left(E_{j}+2 B\right)+2 J^{2}\right] /\left[J\left(E_{j}-J\right)\right], b_{j}=\left(E_{j}+2 B-J\right) /\left(E_{j}-J\right)$, $a_{l}=\left[-\left(E_{l}+J\right)\left(E_{l}-2 B\right)+2 J^{2}\right] /\left[J\left(E_{l}-J\right)\right], b_{l}=\left(E_{l}-2 B-J\right) /\left(E_{l}-J\right), j=2,3,4$, $l=6,7,8$.

The ground state of system (7) is the linear combination of states $\left|\psi_{3}\right\rangle$ and $\left|\psi_{7}\right\rangle$ and is twofold degenerate if $J>0$. The thermal ground state has the probabilities of one half occupying $\left|\psi_{3}\right\rangle$ and one half occupying $\left|\psi_{7}\right\rangle$. The pairwise entanglements at the thermal ground state are

$$
C_{12}=C_{23}=\max \{0, A, B\} \quad C_{13}=\max \{0, C, D\}
$$

where $A=\left|k_{3}^{2} b_{3}+k_{7}^{2} b_{7}\right|-\left[\left(k_{3}^{2} b_{3}^{2}+k_{7}^{2} a_{7}^{2}\right)\left(k_{3}^{2} a_{3}^{2}+k_{7}^{2} b_{7}^{2}\right)\right]^{1 / 2}, B=\left|k_{3}^{2} a_{3} b_{3}+k_{7}^{2} a_{7} b_{7}\right|-\left[\left(k_{3}^{2}+\right.\right.$ $\left.\left.k_{7}^{2} b_{7}^{2}\right)\left(k_{3}^{2} b_{3}^{2}+k_{7}^{2}\right)\right]^{1 / 2}, C=\left|k_{3}^{2} a_{3}+k_{7}^{2} a_{7}\right|-\left|k_{3}^{2} b_{3}^{2}+k_{7}^{2} b_{7}^{2}\right|, D=\left|k_{3}^{2} b_{3}^{2}+k_{7}^{2} b_{7}^{2}\right|-\left[\left(k_{3}^{2}+k_{7}^{2} a_{7}^{2}\right)\right.$ $\left.\left(k_{3}^{2} a_{3}^{2}+k_{7}^{2}\right)\right]^{1 / 2}$. Let us consider two limit cases: (i) the ground state is $\left|\psi_{3}\right\rangle$ and (ii) the ground state is $\left|\psi_{7}\right\rangle$. In the first limit case, the pairwise entanglements are

$$
C_{12}=C_{23}=2 k_{3}^{2}|| a_{3} b_{3}|-| b_{3}|| \quad C_{13}=2 k_{3}^{2}\left|b_{3}^{2}-\right| a_{3}|| .
$$

The entanglements between the $i$ th site and the rest of the other sites are

$$
C_{1 \overline{1}}=C_{3 \overline{3}}=4 k_{3}^{4}\left(1+b_{3}^{2}\right)\left(a_{3}^{2}+b_{3}^{2}\right) \quad C_{2 \overline{2}}=8 k_{3}^{4}\left(1+a_{3}^{2}\right) b_{3}^{2} .
$$

The residual entanglement of the system is $C_{123}=16 k_{3}^{4}\left|a_{3}\right| b_{3}^{2}$ and the global entanglement of the whole system is

$$
Q\left(\left|\psi_{3}\right\rangle\right)=\frac{8}{3} k_{3}^{4}\left[a_{3}^{2}+2 b_{3}^{2}+2 a_{3}^{2} b_{3}^{2}+b_{3}^{4}\right] .
$$

The ground state entanglement versus magnetic impurities is shown in figure 2. In case (ii), one can obtain the same formulae by changing $a_{3}$ and $b_{3}$ into $a_{7}$ and $b_{7}$, respectively. If $J<0$, the ground state is the linear combination of states $\left|\psi_{2}\right\rangle$ and $\left|\psi_{6}\right\rangle$. The entanglement properties can be obtained similarly.

The pairwise entanglement between sites 1 and 2 is the same as that between sites 2 and 3 due to the symmetry. So we only consider the entanglement between sites 1 and 2, and that between sites 1 and 3. After tedious calculation, the pairwise entanglements at finite temperature are

$$
\begin{aligned}
& C_{12}=\frac{2}{Z} \max \{0,|D|-\sqrt{B E},|C|-\sqrt{A F}\} \\
& C_{13}=\frac{2}{Z} \max \{0,|\tilde{D}|-|\tilde{B}|,|\tilde{C}|-\sqrt{\tilde{A} \tilde{F}}\},
\end{aligned}
$$




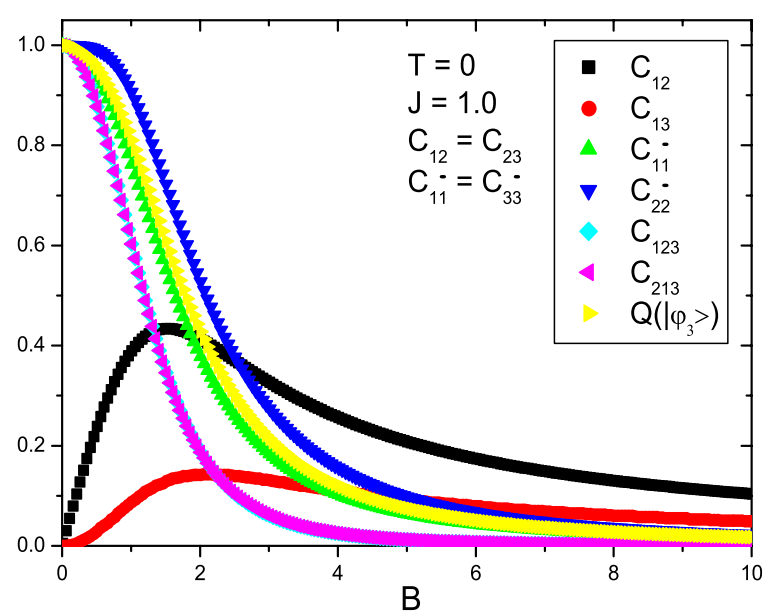

Figure 2. The ground state entanglement in the Ising model with two magnetic impurities. One can see that $C_{123}=C_{213}$, which means that the residual entanglement is independent of permutations of sites.

where $A=0.5 \mathrm{e}^{-\beta E_{1}}+\sum_{i} k_{i}^{2} \mathrm{e}^{-\beta E_{i}}+\sum_{j} k_{j}^{2} a_{j}^{2} \mathrm{e}^{-\beta E_{j}}, B=0.5 \mathrm{e}^{-\beta E_{1}}+\sum_{i} k_{i}^{2} b_{i}^{2} \mathrm{e}^{-\beta E_{i}}+$ $\sum_{j} k_{j}^{2} a_{j}^{2} \mathrm{e}^{-\beta E_{j}}, C=\sum_{l} k_{l}^{2} a_{l} b_{l} \mathrm{e}^{-\beta E_{l}}, D=\sum_{l} k_{l}^{2} b_{l} \mathrm{e}^{-\beta E_{l}}, E=0.5 \mathrm{e}^{-\beta E_{1}}+\sum_{i} k_{i}^{2} a_{i}^{2} \mathrm{e}^{-\beta E_{i}}+$ $\sum_{j} k_{j}^{2} b_{j}^{2} \mathrm{e}^{-\beta E_{j}}, F=\sum_{i} k_{i}^{2} b_{i}^{2} \mathrm{e}^{-\beta E_{i}}+0.5 \mathrm{e}^{-\beta E_{1}}+\sum_{j} k_{j}^{2} \mathrm{e}^{-\beta E_{j}}, \tilde{A}=\sum_{i} k_{i}^{2} \mathrm{e}^{-\beta E_{i}}+$ $\sum_{j} k_{j}^{2} a_{j}^{2} \mathrm{e}^{-\beta E_{j}}, \tilde{B}=\mathrm{e}^{-\beta E_{1}}+\sum_{l} k_{l}^{2} b_{l}^{2} \mathrm{e}^{-\beta E_{l}}, \tilde{C}=-\mathrm{e}^{-\beta E_{1}}+\sum_{l} k_{l}^{2} b_{l}^{2} \mathrm{e}^{-\beta E_{l}}, \tilde{D}=$ $\sum_{l} k_{l}^{2} a_{l} \mathrm{e}^{-\beta E_{l}}, \tilde{F}=\sum_{i} k_{i}^{2} a_{i}^{2} \mathrm{e}^{-\beta E_{i}}+\sum_{j} k_{j}^{2} \mathrm{e}^{-\beta E_{j}}, i=2,3,4, j=6,7,8$ and $l=$ $2,3,4,6,7,8$. The pairwise entanglement between sites 1 and 2 is zero, while the pairwise entanglement between sites 1 and 3 is not equal to zero, which is shown in figure 3 . We find that there exists a critical $B_{\mathrm{cr}}(T)$. There is no entanglement if $B<B_{\mathrm{cr}}(T)$, which means that the heat effect is dominant. The value of critical $B_{\mathrm{cr}}(T)$ increases with increasing temperature. If the temperature is low, this switch effect is not obvious.

\section{Entanglement in the transverse Ising model}

The Hamiltonian of the three-site Ising model with transverse fields is

$$
H=-J\left(\sigma_{1}^{x} \sigma_{2}^{x}+\sigma_{2}^{x} \sigma_{3}^{x}+\sigma_{3}^{x} \sigma_{1}^{x}\right)+B\left(\sigma_{1}^{z}+\sigma_{2}^{z}+\sigma_{3}^{z}\right)
$$

which also means that every site has a magnetic impurity. The eigenstates can be obtained explicitly as

$$
\begin{array}{ll}
\left|\psi_{1}\right\rangle=(|110\rangle-|011\rangle) / \sqrt{2} & \left|\psi_{2}\right\rangle=(|101\rangle-|011\rangle) / \sqrt{2} \\
\left|\psi_{3}\right\rangle=(|100\rangle-|001\rangle) / \sqrt{2} & \left|\psi_{4}\right\rangle=(|010\rangle-|001\rangle) / \sqrt{2} \\
\left|\psi_{5,6}\right\rangle=k_{5,6}\left(a_{5,6}|111\rangle+|001\rangle+|010\rangle+|100\rangle\right) & \\
\left|\psi_{7,8}\right\rangle=k_{7,8}\left(a_{7,8}|000\rangle+|011\rangle+|101\rangle+|110\rangle\right) &
\end{array}
$$

with the eigenvalues $E_{1}=E_{2}=J+B, E_{3}=E_{4}=J-B, E_{5,6}=-J+B \mp$ $2\left[J^{2}+J B+B^{2}\right]^{1 / 2}, E_{7,8}=-J-B \mp 2\left[J^{2}-J B+B^{2}\right]^{1 / 2}$. Here $a_{5,6}=[-J-$ $\left.2 B \pm 2\left(J^{2}+J B+B^{2}\right)^{1 / 2}\right] / J$ and $a_{7,8}=\left[-J+2 B \pm 2\left(J^{2}-J B+B^{2}\right)^{1 / 2}\right] / J$.

The ground state is $\left|\psi_{7}\right\rangle$ no matter $J>0$ or $J<0$. The structure of the ground state $\left|\psi_{7}\right\rangle$ of the transverse field Ising model changes dramatically as the parameter $B$ is varied. When 


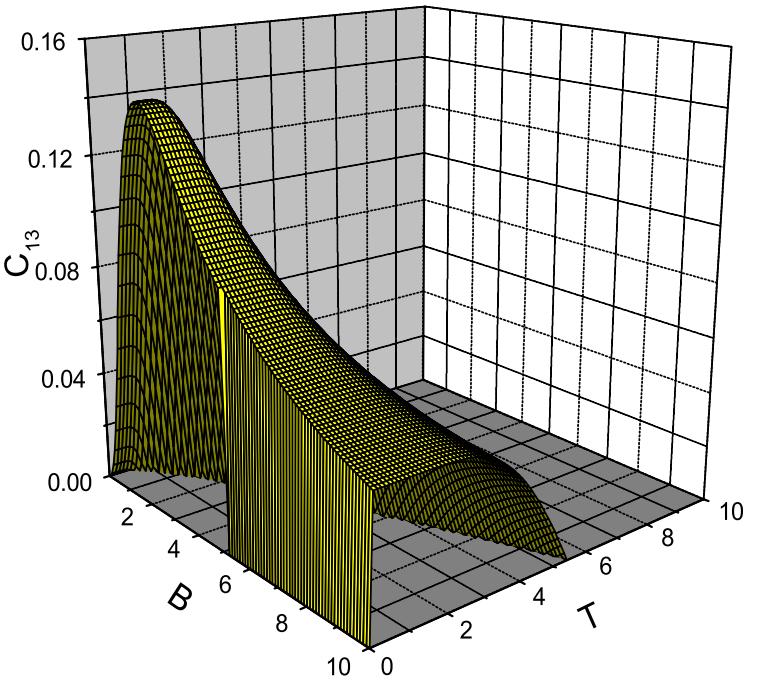

Figure 3. The concurrence $C_{13}$ versus impurities and temperature in the Ising model with two boundary impurities. Here $J=1.0$. There exists a critical $B_{\mathrm{cr}}(T)$ and there is no entanglement if $B<B_{\mathrm{cr}}(T)$, which means that the heat effect is dominant. The value of $B_{\mathrm{cr}}(T)$ increases with increasing temperature. The pairwise entanglement appears only if the magnitude of the impurity is large enough.

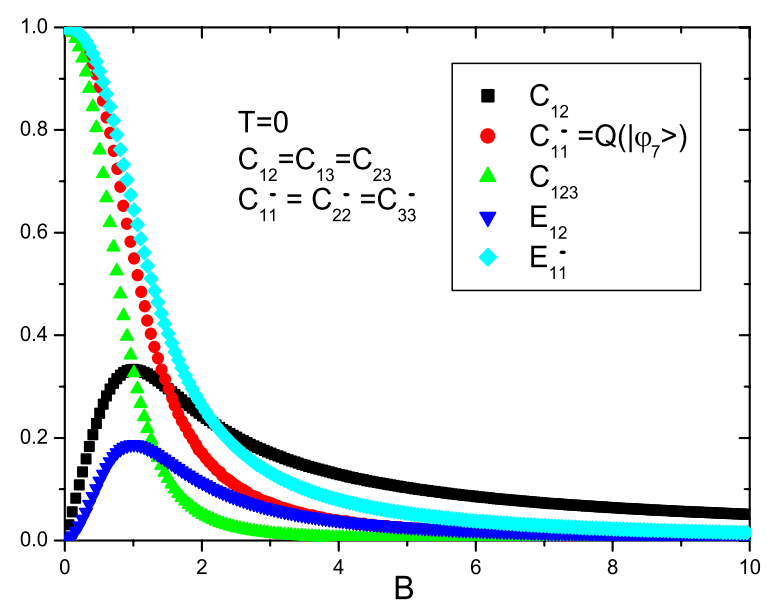

Figure 4. The entanglement at ground state in the transverse Ising model. Here $J=1.0$. There is no entanglement if $B=0$. However, if there exists an infinitesimal transverse field, the tangle $C_{1 \overline{1}}$ (or $E_{1 \overline{1}}$ ), the residual entanglement $C_{123}$ and the global entanglement $Q\left(\left|\psi_{7}\right\rangle\right.$ ) reach their maxima immediately. Then, the tangle, residual entanglement and global entanglement decrease with increasing transverse field, while the behaviour of concurrence is very different from that, and concurrence reaches its maximum at the point $B=1$. Therefore, different measures of entanglement reach their maxima at different magnitudes of transverse field.

$B / J$ approaches 0 , the ground state becomes a product of spins pointing in the $x$ direction, and is not entangled. When $B / J$ tends to infinity, the ground state approaches again a product of spins pointing in the $z$ direction, and is also not entangled. The pairwise entanglement at finite $B / J$ can be measured by concurrence as $C_{12}=2 k_{7}^{2}|1-| a_{7}||$. Due to the periodic boundary 
conditions, any pairwise entanglements of the system are equal. The entanglement of site 1 with the rest of the other sites is $C_{1 \overline{1}}=8 k_{7}^{4}\left(a_{7}^{2}+1\right)$, and the residual entanglement of the system is $C_{123}=16 k_{7}^{4}\left|a_{7}\right|$. The global entanglement of the whole system reads $Q\left(\left|\psi_{7}\right\rangle\right)=C_{1 \overline{1}}$ due to the translation invariant. In order to analogize the different measures of entanglement, we also calculate the von Neumann entropy, another measure of entanglement. The von Neumann entropy of sites 1 and 2 can be obtained from the concurrence as

$$
\begin{aligned}
& E_{12}\left(C_{12}\right)=h\left(\left[1+\sqrt{1-C_{12}^{2}}\right] / 2\right) \\
& h(x)=-x \log _{2} x-(1-x) \log _{2}(1-x) .
\end{aligned}
$$

The von Neumann entropy calculated from the reduced density matrix $\rho_{1}$ is employed to measure the entanglement of states on the first site with that on the remaining sites,

$$
E_{1 \overline{1}}=-k_{7}^{2}\left(a_{7}^{2}+1\right) \log _{2} k_{7}^{2}\left(a_{7}^{2}+1\right)-2 k_{7}^{2} \log _{2} 2 k_{7}^{2} .
$$

It exhibits the correlations between a local state and the other part of the system. From figure 4, we find that the concurrence $C_{12}$ has a maximum value at the point $B=1$. Note that if the total lattice number of the chain is more than 3 , the maximum of concurrence will appear before but near the point $B=1$, while the tangle $C_{1 \overline{1}}$ is a monotonically decreasing function with increasing $B$, whose behaviour is very different from that of the concurrence. It is argued that the entanglement can be used to describe the quantum phase transitions and the entanglement quantification may reach its maximum at the critical point [12]. If this argument is right, the critical point of the quantum phase transitions should correspond to the maximum of total entanglement because both of them describe the global properties of the system. Therefore, if one uses the concurrence to measure the entanglement, the pairwise entanglement may not arrive at its maximum at the critical point because it is not the total entanglement of the system. Note that different measures of entanglement may reach their maxima at different values of intrinsic parameters in the system.

The concurrence at finite $T$ is $C_{12}=2 \max \{0,|D|-\sqrt{B E},|C|-\sqrt{A F}\} / Z$ where $A=\mathrm{e}^{-\beta E_{3}}+\sum_{j} k_{j}^{2} \mathrm{e}^{-\beta E_{j}}+\sum_{l} k_{l}^{2} a_{l}^{2} \mathrm{e}^{-\beta E_{l}}, B=\mathrm{e}^{-\beta E_{1}}+0.5 \mathrm{e}^{-\beta E_{3}}+\sum_{j, l} k_{j, l}^{2} \mathrm{e}^{-\beta E_{j, l}}, C=$ $-0.5 \mathrm{e}^{-\beta E_{1}}+\sum_{j} k_{j}^{2} \mathrm{e}^{-\beta E_{j}}+\sum_{l} k_{l}^{2} \mathrm{e}^{-\beta E_{l}}, D=\sum_{j, l} k_{j, l}^{2} a_{j, l} \mathrm{e}^{-\beta E_{j, l}}, E=\sum^{2} 0.5 \mathrm{e}^{-\beta E_{1,3}}+$ $\sum_{j, l} k_{j, l}^{2} \mathrm{e}^{-\beta E_{j, l}}, F=0.5 \mathrm{e}^{-\beta E_{1}}+\sum_{j} k_{j}^{2} a_{j}^{2} \mathrm{e}^{-\beta E_{j}}+\sum_{l} k_{l}^{2} \mathrm{e}^{-\beta E_{l}}, j=5,6, l=7,8$. The behaviour of thermal pairwise entanglement is shown in figure 5 .

Now, we can safely argue that at zero temperature, there is no entanglement if $B=0$. If there exists an impurity, the pairwise entanglement appears and the concurrence is not equal to zero. At finite temperature, we find a switch effect of the entanglement. The heat effect is dominant and there is no pairwise entanglement if the magnitude of the impurity is small. The impurity effect is dominant and the pairwise entanglement appears only if the impurity is large enough. The competition of temperature and impurities leads to a critical $B_{\mathrm{cr}}(T)$. The impurities can lead to pairwise entanglement only in the region of $B>B_{\mathrm{cr}}(T)$. The value of $B_{\mathrm{cr}}(T)$ increases with increasing temperature. It is worth pointing out that all excited states have contributions to the entanglement at finite temperature. The eigenstate of the system at thermal equilibrium is a mixed state. The concurrence of the two-qubit mixed state $\rho$ is defined to be a minimum over all possible pure-state decompositions of mixed state. The concurrence of different pure states of $\left\{p_{i},\left|\psi_{i}\right\rangle\right\}$ may cancel and leads to the switch effect. In the region of $B<B_{\mathrm{cr}}(T)$, the mixed state pairwise thermal entanglement is zero, but one can check that every excited state of thermal equilibrium has nonzero pairwise entanglement. 


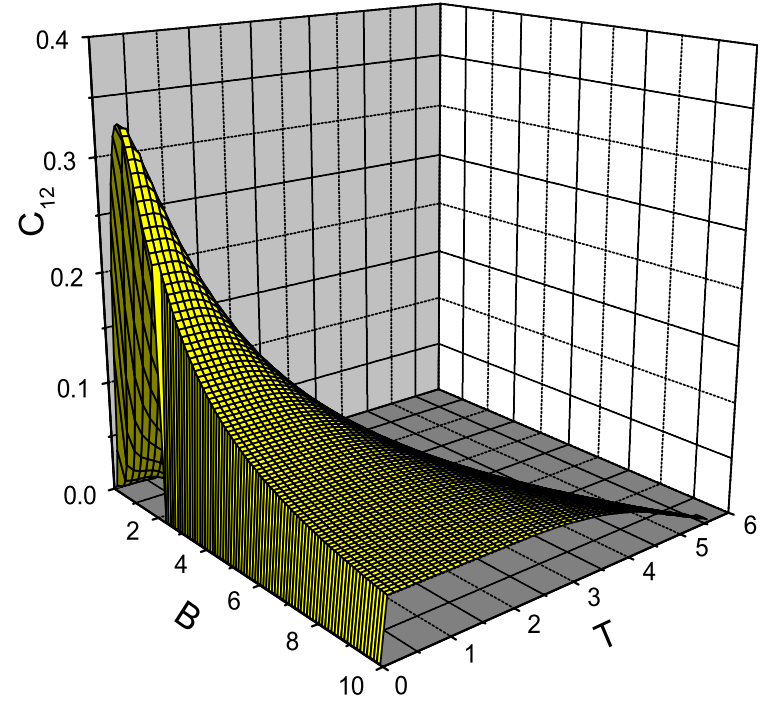

Figure 5. The concurrence $C_{12}$ versus impurities and temperature in the transverse Ising model. Here $J=1.0$. There exists a critical $B_{\mathrm{cr}}(T)$ and there is no entanglement if $B<B_{\mathrm{cr}}(T)$, which means that the heat effect is dominant. The value of $B_{\mathrm{cr}}(T)$ increases with increasing temperature.

\section{Entanglement in the Ising model with two boundary impurities}

In this section, we consider the three-site Ising model with open boundary conditions, which also means that there are two magnetic impurities on the two boundaries. The impurities break the translation invariant of the system. The Hamiltonian is

$$
H=-J\left(\sigma_{1}^{x} \sigma_{2}^{x}+\sigma_{2}^{x} \sigma_{3}^{x}\right)+B_{1} \sigma_{1}^{z}+B_{3} \sigma_{3}^{z} .
$$

The eigenstates of system (16) are

$$
\begin{aligned}
& \left|\psi_{i}\right\rangle=k_{i}\left(|000\rangle+a_{i}|011\rangle+b_{i}|101\rangle+c_{i}|110\rangle\right) \\
& \left|\psi_{j}\right\rangle=k_{j}\left(|111\rangle+\tilde{a}_{j}|100\rangle+\tilde{b}_{j}|010\rangle+\tilde{c}_{j}|001\rangle\right)
\end{aligned}
$$

where $a_{i}=-\left(E_{i}-B_{1}+B_{3}\right)\left(E_{i}+B_{1}+B_{3}\right) /\left(2 J E_{i}\right), b_{i}=\left(E_{i}+B_{1}+B_{3}\right) /\left(E_{i}-B_{1}-B_{3}\right)$, $c_{i}=-\left(E_{i}+B_{1}+B_{3}\right)\left(E_{i}+B_{1}-B_{3}\right) /\left(2 J E_{i}\right), \tilde{a}_{j}=-2 J E_{j} /\left[\left(E_{j}-B_{1}+B_{3}\right)\left(E_{j}+B_{1}+B_{3}\right)\right]$, $\tilde{b}_{j}=\left(E_{j}-B_{1}-B_{3}\right) /\left(E_{j}+B_{1}+B_{3}\right), \tilde{c}_{j}=-2 J E_{j} /\left[\left(E_{j}+B_{1}+B_{3}\right)\left(E_{j}+B_{1}-B_{3}\right)\right], i=$ $1, \ldots, 4, j=5, \ldots, 8, k_{i, j}$ are the normalized coefficients and $E_{i, j}$ are the corresponding eigenvalues

$$
\begin{aligned}
& E_{1,5}=-E_{2,6}=\left|\sqrt{B_{1}^{2}+J^{2}}+\sqrt{B_{3}^{2}+J^{2}}\right| \\
& E_{3,7}=-E_{4,8}=\left|\sqrt{B_{1}^{2}+J^{2}}-\sqrt{B_{3}^{2}+J^{2}}\right| .
\end{aligned}
$$

The ground state is a linear combination of states $\left|\psi_{2}\right\rangle$ and $\left|\psi_{6}\right\rangle$. The thermal ground state has the probabilities one half occupying $\left|\psi_{2}\right\rangle$ and one half occupying $\left|\psi_{6}\right\rangle$. All the concurrences at the thermal ground state are zero. If the ground state is $\left|\psi_{2}\right\rangle$, the pairwise entanglements are

$$
C_{12}=C_{23}=2 k_{2}^{2}|| a_{2} b_{2}|-| c_{2}|| \quad C_{13}=0 .
$$

We see that the pairwise entanglement between two boundaries is zero. This is because the directions of boundary impurities are the same. The tangles between the $i$ th site and the rest 


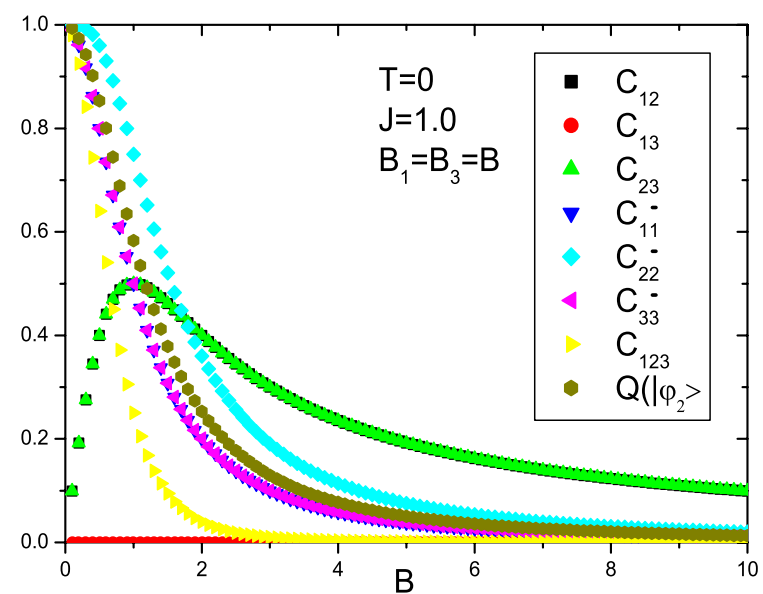

Figure 6. The ground state entanglement in the Ising model with two boundary impurities. $C_{12}=C_{23}, C_{13}=0, C_{1 \overline{1}}=C_{3 \overline{3}}$. There is no entanglement if $B=0$. The tangle, residual entanglement and global entanglement appear and reach their maxima immediately if there exist infinitesimal boundary impurities and decrease with increasing magnitude of impurity. The concurrences $C_{12}$ and $C_{23}$ reach their maxima at the point $B=1.0$.

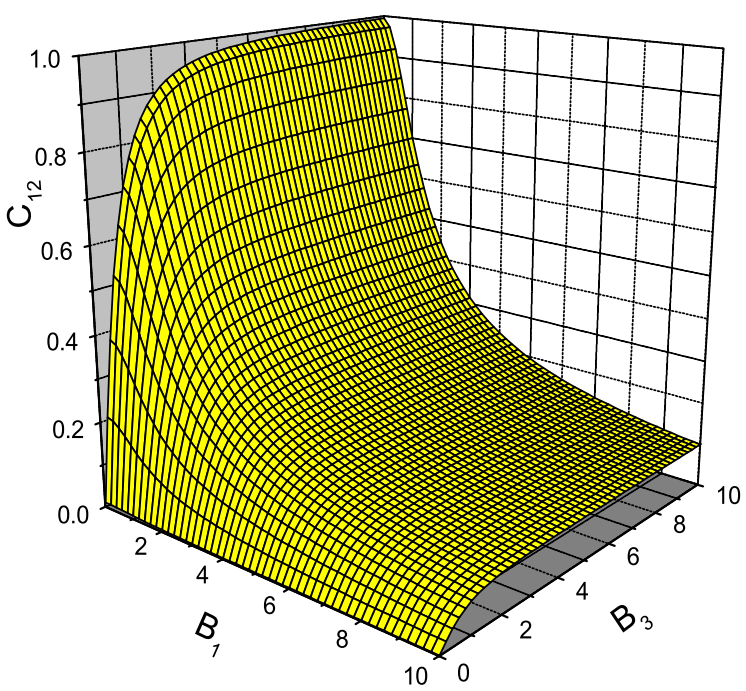

Figure 7. The concurrence $C_{12}$ versus two boundary impurities at the ground state in the Ising model. It is also the curve of concurrence $C_{23}$ after exchanging $B_{1}$ and $B_{3}$ because the system is unchanged after this operation. Here $J=1.0$.

of the other sites are

$$
C_{1 \overline{1}}=C_{3 \overline{3}}=4 k_{2}^{4}\left(1+a_{2}^{2}\right)\left(b_{2}^{2}+c_{2}^{2}\right) \quad C_{2 \overline{2}}=4 k_{2}^{4}\left(1+b_{2}^{2}\right)\left(a_{2}^{2}+c_{2}^{2}\right) .
$$

The residual entanglement is $C_{123}=16 k_{2}^{4}\left|a_{2} b_{2} c_{2}\right|$. The global entanglement of the whole system reads

$$
Q\left(\left|\psi_{2}\right\rangle\right)=\frac{8}{3} k_{2}^{4}\left(a_{2}^{2}+b_{2}^{2}+c_{2}^{2}+a_{2}^{2} b_{2}^{2}+b_{2}^{2} c_{2}^{2}+a_{2}^{2} c_{2}^{2}\right) .
$$

The entanglement properties are shown in figure 6 . The boundary impurities effect on the concurrence $C_{12}$ is shown in figure 7 . 


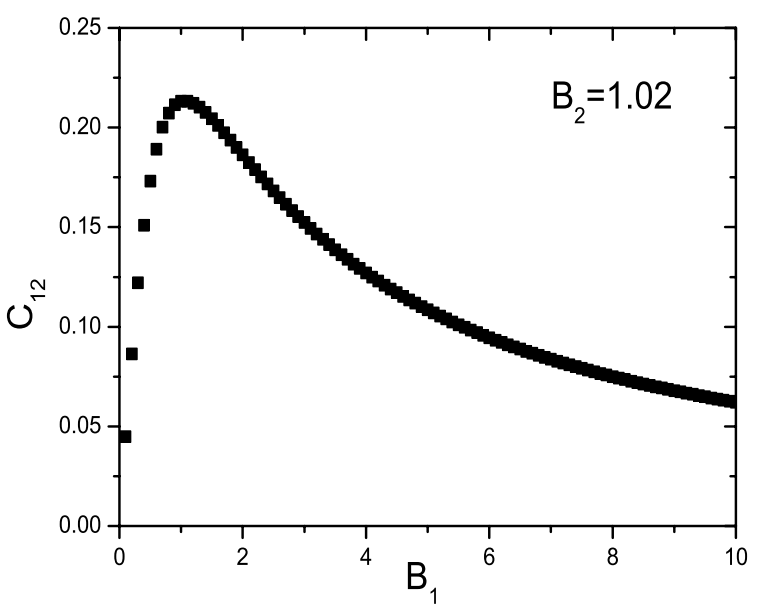

Figure 8. The concurrence $C_{12}$ versus impurity at the thermal ground state in the Ising model with one free boundary. The concurrence arrives at its maximum at the critical point $B_{1}=1.0$.

Any pairwise entanglement at finite temperature is zero. This is because the eigenstate of the system at thermal equilibrium is a mixed state and the concurrences of different pure states of the mixed state may cancel each other. One can check that the concurrence, tangle, residual and global entanglement of every excited state are not equal to zero.

\section{Entanglement in the Ising model with one free boundary}

Now, we consider the three-site Ising model with two neighbour impurities using free boundary conditions. The Hamiltonian is

$$
H=-J\left(\sigma_{1}^{x} \sigma_{2}^{x}+\sigma_{2}^{x} \sigma_{3}^{x}\right)+B_{1} \sigma_{1}^{z}+B_{2} \sigma_{2}^{z} .
$$

Here we put one impurity in the bulk and one on the boundary, while the other boundary is free. There is only one impurity in the bulk and two boundaries are free if $B_{1}=0$. The corresponding eigenstates of system (22) are

$$
\begin{aligned}
& \left|\psi_{i}\right\rangle=k_{i}\left(|000\rangle+a_{i}|011\rangle+b_{i}|101\rangle+c_{i}|110\rangle\right) \\
& \left|\psi_{j}\right\rangle=k_{j}\left(|111\rangle+\tilde{a}_{j}|100\rangle+\tilde{b}_{j}|010\rangle+\tilde{c}_{j}|001\rangle\right)
\end{aligned}
$$

where $a_{i}=-\left(E_{i}-B_{1}-B_{2}\right)\left(E_{i}+B_{1}+B_{2}\right) /\left(2 J\left(E_{i}-B_{2}\right)\right), b_{i}=\left(E_{i}+B_{1}+B_{2}\right) /\left(E_{i}-B_{1}+B_{2}\right)$, $c_{i}=-\left(E_{i}+B_{1}+B_{2}\right)\left(E_{i}+B_{1}-B_{2}\right) /\left(2 J\left(E_{i}-B_{2}\right)\right), \tilde{a}_{j}=-\left(E_{j}+B_{1}+B_{2}\right)\left(E_{j}-B_{1}-\right.$ $\left.B_{2}\right) /\left(2 J\left(E_{i}+B_{2}\right)\right), \tilde{b}_{j}=\left(E_{j}-B_{1}-B_{2}\right) /\left(E_{j}+B_{1}-B_{2}\right), \tilde{c}_{j}=\left(E_{j}-B_{1}-B_{2}\right)\left(E_{j}-B_{1}+\right.$ $\left.B_{2}\right) /\left(2 J\left(E_{i}+B_{2}\right)\right), i=1,2,3,4, j=5,6,7,8, k_{i, j}$ are the normalized coefficients and $E_{i, j}$ are the corresponding eigenvalues

$$
\begin{aligned}
& E_{1,5}=-E_{2,6}=\left[B_{1}^{2}+B_{2}^{2}+2 J^{2}+2 \sqrt{B_{1}^{2} B_{2}^{2}+B_{1}^{2} J^{2}+J^{4}}\right]^{\frac{1}{2}} \\
& E_{3,7}=-E_{4,8}=\left[B_{1}^{2}+B_{2}^{2}+2 J^{2}-2 \sqrt{B_{1}^{2} B_{2}^{2}+B_{1}^{2} J^{2}+J^{4}}\right]^{\frac{1}{2}} .
\end{aligned}
$$

The ground state of the system is the linear combination of states $\left|\psi_{2}\right\rangle$ and $\left|\psi_{6}\right\rangle$. The thermal ground state is mixed by $\left|\psi_{2}\right\rangle$ and $\left|\psi_{6}\right\rangle$ with equal probabilities and the density matrix 


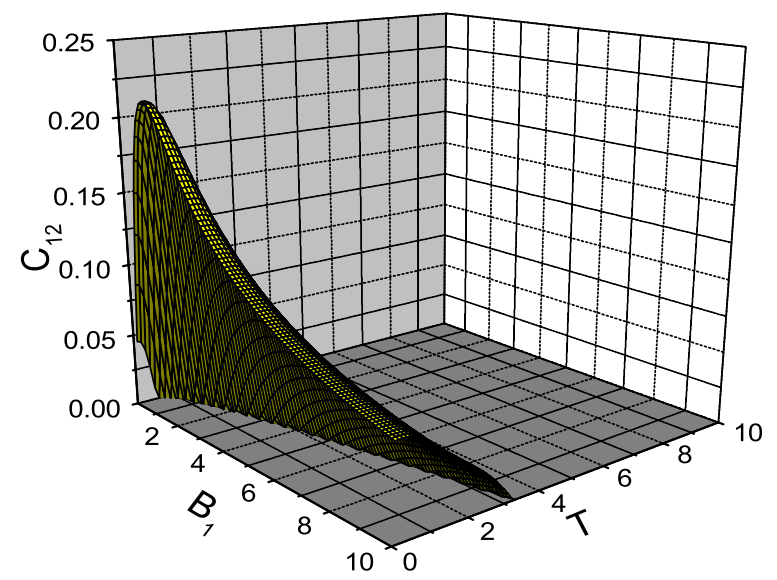

Figure 9. The concurrence $C_{12}$ versus temperature and impurity in the Ising model with one free boundary. Here $J=1.0$ and $B_{2}=1.02$.

is

$$
\rho_{\text {ground }}=\frac{1}{2}\left|\psi_{2}\right\rangle\left\langle\psi_{2}\left|+\frac{1}{2}\right| \psi_{6}\right\rangle\left\langle\psi_{6}\right|
$$

From the density matrix (25), we have $C_{12}=\left|k_{2}^{2} c_{2}+k_{6}^{2} \tilde{c}_{6}\right|-\left[\left(k_{2}^{2} a_{2}^{2}+k_{6}^{2} \tilde{b}_{6}^{2}\right)\left(k_{2}^{2} b_{2}^{2}+k_{6}^{2} \tilde{a}_{6}^{2}\right)\right]^{1 / 2}$ and $C_{13}=C_{23}=0$. The concurrence $C_{12}$ versus boundary impurity is shown in figure 8 . The $C_{12}$ arrives at its maximum at the critical point $B_{1}=1.0$. The pairwise entanglements at finite temperature are $C_{12}=2 \max \{0,|D|-\sqrt{B E},|C|-\sqrt{A F}\} / Z$ and $C_{13}=C_{23}=0$, where $A=\sum_{i} k_{i}^{2} \mathrm{e}^{-\beta E_{i}}+\sum_{j} k_{j}^{2} \tilde{c}_{j}^{2} \mathrm{e}^{-\beta E_{j}}, B=\sum_{i} k_{i}^{2} a_{i}^{2} \mathrm{e}^{-\beta E_{i}}+$ $\sum_{j} k_{j}^{2} \tilde{b}_{j}^{2} \mathrm{e}^{-\beta E_{j}}, C=\sum_{i} k_{i}^{2} a_{i} b_{i} \mathrm{e}^{-\beta E_{i}}+\sum_{j} k_{j}^{2} \tilde{a}_{j} \tilde{b}_{j} \mathrm{e}^{-\beta E_{j}}, D=\sum_{i} k_{i}^{2} c_{i} \mathrm{e}^{-\beta E_{i}}+\sum_{j} k_{j}^{2} \tilde{c}_{j}$ $\mathrm{e}^{-\beta E_{j}}, E=\sum_{i} k_{i}^{2} b_{i}^{2} \mathrm{e}^{-\beta E_{i}}+\sum_{j} k_{j}^{2} \tilde{a}_{j}^{2} \mathrm{e}^{-\beta E_{j}}, F=\sum_{i} k_{i}^{2} c_{i}^{2} \mathrm{e}^{-\beta E_{i}}+\sum_{j} k_{j}^{2} \mathrm{e}^{-\beta E_{j}}, i=$ $1,2,3,4, j=5,6,7,8$. The concurrence $C_{12}$ versus temperature and boundary field is shown in figure 9 . Note that the concurrences $C_{13}$ and $C_{23}$ are 0 both at the thermal ground state and at the thermal equilibrium state.

\section{Conclusion}

In summary, we study the magnetic impurities effect on entanglement in the Ising model with both periodic and open boundary conditions, and obtain analytic expressions for the measures of entanglement. The ground state and excited states of the system possess remarkable entanglement properties. The relations between pairwise entanglement and magnetic impurities and temperature are obtained. We find that the entanglement properties of the system are dependent on the location of impurities. Different pairs of sites have different entanglement properties. The impurities and symmetry broken in the system can enhance some pairwise entanglement. Therefore, if one wants to enhance the entanglement of some pairs, good methods are doping with impurities or breaking the symmetry of the system.

\section{Acknowledgment}

XRW is supported by UGC, Hong Kong. YW is supported by the NSF of China. He also thanks the Physics Department of HKUST for their hospitality during his visit. 


\section{References}

[1] Bennett C H and DiVincenzo D P 2000 Nature 404247

[2] Bennett C H, Brassard G, Crepeau C, Jozsa R, Peres A and Wootters W K 1993 Phys. Rev. Lett. 701895

[3] Bennett C H and Wiesner S J 1992 Phys. Rev. Lett. 692881

[4] Ekert A 1991 Phys. Rev. Lett. 67661

[5] Gunlycke D, Bose S, Kendon V M and Vedral V 2001 Phys. Rev. A 64042302

[6] Nielsen M A 2000 Preprint quant-ph/0011036

[7] Wang X 2001 Phys. Rev. A 64012313

[8] Wang X 2001 Phys. Lett. A 281101

[9] Zhou L, Song H S, Guo Y Q and Li C 2003 Phys. Rev. A 68024301

[10] Sachdev S 1999 Quantum Phase Transitions (Cambridge: Cambridge University Press)

[11] Osterloh A, Amico L, Falci G and Fazio R 2002 Nature 416608

[12] Osborne T J and Nielsen M A 2002 Phys. Rev. A 66032110

[13] Vidal G, Latorre J I, Rico E and Kitaev A 2003 Phys. Rev. Lett. 90227902

[14] Osenda O, Huang Z and Kais S 2003 Phys. Rev. A 67062321

[15] Korepin V E 2004 Phys. Rev. Lett. 92096402

[16] Arnesen M C, Bose S and Vedral V 2001 Phys. Rev. Lett. 87017901

[17] Verstraete F, Martin-Delgado M A and Cirac J I 2004 Phys. Rev. Lett. 92087201

[18] Pachos J K and Plenio M B 2004 Phys. Rev. Lett. 93056402

[19] Hill S and Wootters W K 1997 Phys. Rev. Lett. 785022

Wootters W K 1998 Phys. Rev. Lett. 802245

[20] Coffman V, Kundu J and Wootters W K 2000 Phys. Rev. A 61052306

[21] Meyer D A and Wallach N R 2002 J. Math. Phys. 434273

[22] Osborne T J 2002 Preprint quant-ph/0203087 